\author{
Stephen M. Klisch ${ }^{1}$ \\ Ph.D. Candidate, \\ Department of Mechanical Engineering, \\ University of California, \\ Berkeley, CA \\ Jeffrey C. Lotz \\ Associate Professor and Director, \\ Orthopædic Bioengineering Laboratory, \\ Department of Orthopædic Surgery, \\ University of California, \\ San Francisco, CA 94143
}

\section{A Special Theory of Biphasic Mixtures and Experimental Results for Human Annulus Fibrosus Tested in Confined Compression}

\begin{abstract}
A finite deformation mixture theory is used to quantify the mechanical properties of the annulus fibrosus using experimental data obtained from a confined compression protocol. Certain constitutive assumptions are introduced to derive a special mixture of an elastic solid and an inviscid fluid, and the constraint of intrinsic incompressibility is introduced in a manner that is consistent with results obtained for the special theory. Thirty-two annulus fibrosus specimens oriented in axial $(n=16)$ and radial $(n=16)$ directions were obtained from the middle-lateral portion of intact intervertebral discs from human lumbar spines and tested in a stress-relaxation protocol. Material constants are determined by fitting the theory to experimental data representing the equilibrium stress versus stretch and the surface stress time history curves. No significant differences in material constants due to orientation existed, but significant differences existed due to the choice of theory used to fit the data. In comparison with earlier studies with healthy annular tissue, we report a lower aggregate modulus and a higher initial permeability constant. These differences are explained by the choice of reference configuration for the experimental studies. [S0148-0731(00)01002-5]
\end{abstract}

\section{Introduction}

Several studies have demonstrated that intervertebral disc degeneration and mechanical failure of the annulus fibrosus by nuclear prolapse occur simultaneously in patients with low back pain $[1,2]$ and that annular degeneration is the product of a cellular remodeling response to alterations in tissue stress $[3,4]$. These observations suggest that the quantification of the mechanical properties and the prediction of the in vivo stresses for the intervertebral disc may lead to an improved understanding of the complex interactions between mechanical loading, degeneration, and structural failure. The intervertebral disc tissue is composed of three major constituents: water, proteoglycan, and collagen. Finite-element studies suggest that the healthy disc simultaneously experiences high tensile and compressive strains under physiological loading [5]. This loading induces fluid flow into and through the disc, which is important for nutritional purposes and maintaining the biological composition [6,7]. Thus, it is important that constitutive models for disc tissue accurately represent large deformations and fluid flow through the proteoglycan-collagen solid matrix.

Mow et al. [8] used the theory of Craine et al. [9] with the constraint of intrinsic incompressibility [10] to derive the linear biphasic mixture theory. This theory has successfully described the creep and stress-relaxation behavior of various biological tissues, including articular cartilage $[8,11,12]$ and annulus fibrosus $[13,14]$. The development is based upon modern mixture theory, which was developed by authors such as Truesdell and Toupin [15], Green and Naghdi [16,17], Bowen [18], and Müller [19]. Because large deformations were predicted in the solid matrix, a

${ }^{1}$ Present address: Mechanical and Aerospace Engineering Department, University of California, San Diego, 9500 Gilman Drive, La Jolla, CA 92092.

Contributed by the Bioengineering Division for publication in the JOURNAL OF BIOMECHANICAL ENGINEERING. Manuscript received by the Bioengineering Division October 13, 1998; revised manuscript received November 30, 1999. Associate Technical Editor: L. A. Taber. finite deformation theory was subsequently used to derive nonlinear constitutive restrictions [12]. Several simplifying assumptions were made to derive a "special" theory, which serves as the basis for several experimental studies $[14,20,21]$. In particular, it was assumed that the solid and the fluid free energy functions were equal and, apparently, that the partial derivative of the fluid free energy with respect to the fluid density vanishes. Several other biomechanical studies have arrived at a similar set of constitutive equations: Holmes [22] proposed a nonlinear theory but made no mention of a fluid free energy or a determinate fluid stress; Oomens et al. [23] neglected the determinate fluid stress term that naturally appeared in their development; and Cohen [24] assumed that the fluid free energy function is constant. However, the consequence of these constitutive assumptions has not been evaluated in any theoretical or experimental study in the biomechanics literature.

In an attempt to address these issues, we employed an alternative approach for obtaining a special mixture theory and utilized new experimental data to quantify the effect of a particular fluid free energy function on the numerical solution to a stressrelaxation protocol. The primary aims of the present work were to introduce a special theory of an intrinsically incompressible mixture of an elastic solid and an inviscid fluid and to quantify the mechanical properties of the annulus using experimental data from a confined compression protocol. In comparison with earlier studies with healthy annular tissue that used a different reference configuration, we report a lower aggregate modulus and a higher initial permeability constant. Thus, a third aim was to explain these differences and to suggest that our choice of reference configuration is more consistent with an assumption made in the constitutive development.

\section{Theory}

In the present paper, the superscripts $s$ and $f$ will refer to the solid and the fluid constituents, respectively. We adopt an approach based on the theory of Krishnaswamy and Batra [25] for a 
mixture of an elastic solid and a viscous fluid. In this approach, a homothermal quasi-static process (HQSP) is constructed, which is assumed to be reversible, and an entropy prescription is obtained. We make two modifications to this theory. First, we consider a mixture of an elastic solid and an inviscid fluid; thus, all viscous properties of the mixture are modeled by the momentum supply. This assumption may be valid for the slow flow of fluid through the solid, which is a characteristic of our experimental protocol. Second, we follow the suggestion of Atkin and Craine [26], who derived a special theory of an isotropic elastic solid and a viscous fluid. In particular, we assume: (1) the "equilibrium state" variables do not include $\mathbf{G}^{s}=\operatorname{grad} \mathbf{F}^{s}$ (where $\mathbf{F}^{s}$ is the deformation gradient tensor for the solid constituent) or $\operatorname{grad} \rho^{f}$ (where $\rho^{f}$ is the apparent fluid density); and (2) the partial stresses and the momentum supply are linear functions of the relative velocity a $=\mathbf{v}^{s}-\mathbf{v}^{f}$ (where $\mathbf{v}^{\alpha}$ is the velocity of the $\alpha$ constituent). The first assumption is made in an attempt to simplify the constitutive equations. The second assumption is valid for low values of the relative velocity. The derivation of the results that follow are presented in full by Klisch [27]; the reader may consult Atkin and Craine [28] for a detailed derivation and discussion of the balance equations.

Thus, we define the quantity $\boldsymbol{\Delta}$ as $\left\{\mathbf{F}^{s}, \rho^{f}, \theta\right\}$ and write

$$
\begin{gathered}
\mathbf{T}^{\alpha}={ }_{0} \hat{\mathbf{T}}^{\alpha}(\boldsymbol{\Delta})+{ }_{e} \hat{\mathbf{T}}^{\alpha}(\boldsymbol{\Delta}) \mathbf{a} \\
\boldsymbol{\pi}^{\alpha}={ }_{0} \hat{\boldsymbol{\pi}}^{\alpha}(\boldsymbol{\Delta})+{ }_{e} \hat{\boldsymbol{\pi}}^{\alpha}(\boldsymbol{\Delta}) \mathbf{a} \\
\psi^{\alpha}={ }_{0} \hat{\psi}^{\alpha}(\boldsymbol{\Delta})+{ }_{e} \hat{\psi}^{\alpha}(\boldsymbol{\Delta}, \mathbf{a})
\end{gathered}
$$

where $\theta$ is the common mixture temperature, $\mathbf{T}^{\alpha}$ is the partial Cauchy stress tensor, $\boldsymbol{\pi}^{\alpha}$ is the internal momentum supply, and $\psi^{\alpha}$ is the partial Helmholtz free energy function. We define the Helmholtz free energy function of the mixture as

$$
\rho \psi=\rho^{s} \psi^{s}+\rho^{f} \psi^{f}
$$

where $\rho=\rho^{s}+\rho^{f}$ is the mixture density. From the mixture energy equation for a HQSP we obtain the result

$$
\frac{\partial_{0} \hat{\psi}^{s}}{\partial \rho^{f}}=\frac{\partial_{0} \hat{\psi}^{f}}{\partial \mathbf{F}^{s}}=0
$$

which is the same result obtained by Atkin and Craine [26]. Thus, we have verified the constitutive restrictions, Eq. (5), using the present theory as opposed to the theory used by Atkin and Craine [26] in which all constitutive restrictions were derived from an entropy inequality. As a consequence of Eq. (5), the constitutive restrictions that we obtain from the mixture energy equation for a HQSP reduce to

$$
\begin{gathered}
{ }_{0} \mathbf{T}^{s}=\rho^{s} \frac{\partial_{0} \hat{\psi}^{s}}{\partial \mathbf{F}^{s}} \mathbf{F}^{s^{T}} \\
{ }_{0} \mathbf{T}^{f}=-\rho^{f^{2}} \frac{\partial_{0} \hat{\psi}^{f}}{\partial \rho^{f}} \mathbf{I} \\
{ }_{0} \boldsymbol{\pi}=\mathbf{0} .
\end{gathered}
$$

The Clausius-Duhem inequality provides additional restrictions; those important for the present application are

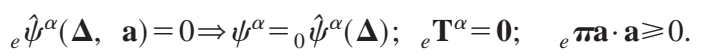

Furthermore, due to the assumptions made in the special theory, from Eqs. (3), (5), and (9), it is apparent that $\psi$ obeys the additive decomposition

$$
\rho \psi\left(\mathbf{F}^{s}, \quad \rho^{f}\right)=\rho^{s} \psi^{s}\left(\mathbf{F}^{s}\right)+\rho^{f} \psi^{f}\left(\rho^{f}\right) .
$$

The approach taken here to define a constrained special mixture is based on a geometric treatment of internally constrained mix- tures of thermoelastic continua [29]. Specifically, we consider an internal constraint of the form

$$
\phi\left(\mathbf{F}^{s}, \rho^{f}\right)=0
$$

where $\phi$ is a sufficiently smooth scalar-valued function defined for the subset of the 10-dimensional space $\mathcal{E}$ for which $\operatorname{det} \mathbf{F}^{\alpha}$ $>0$ and $\rho^{f}>0$. This constraint represents a 9-dimensional constraint manifold $\mathcal{S}$. Upon assuming that the constraint remains invariant under superposed rigid body motions, Eq. (11) may be written in the objective form $\hat{\phi}\left(\mathbf{C}^{s}, \rho^{f}\right)=0$ where $\mathbf{C}^{s}$ is the right Cauchy-Green tensor; however, we use the more primitive form, Eq. (11), to be consistent with Klisch [29]. The constraint, Eq. (11), is considered while the material point $\mathbf{X}^{f}$ is held fixed, although it can also be considered while the material point $\mathbf{X}^{s}$ or the spatial point $\mathbf{x}$ are held fixed. A normal to $\mathcal{S}$ is given by $\mathbf{n}$ $=\left(\partial \phi / \partial \mathbf{F}^{s}, \partial \phi / \partial \rho^{f}\right)$ while a tangent to $\mathcal{S}$ is given by $\mathbf{t}$ $=\left(D^{f} \mathbf{F}^{s} / D t, D^{f} \rho^{f} / D t\right)$ where $D^{\alpha}(\cdot) / D t$ is the material time derivative following the $\alpha$ constituent. Hence, for any process satisfying the constraint it is necessary that $\mathbf{n} \cdot \mathbf{t}=0$. A constrained special mixture $m^{\prime}$ is constructed from an unconstrained special mixture $m$ by first assuming that $m^{\prime}$ inherits the quantities $\left(\psi_{m}^{\alpha}, \mathbf{T}_{m}^{\alpha}, \boldsymbol{\pi}_{m}\right)$ for $m$ when evaluated on the constraint manifold $\mathcal{S}$. Using the approach of Klisch [29], it is found that the partial stresses and momentum supply for $m^{\prime}$ must satisfy

$$
\begin{gathered}
\mathbf{T}_{m^{\prime}}^{s}=\mathbf{T}_{m}^{s}+p \frac{\partial \phi}{\partial \mathbf{F}^{s}} \mathbf{F}^{s^{T}} \\
\mathbf{T}_{m^{\prime}}^{f}=\mathbf{T}_{m}^{f}+p \frac{\partial \phi}{\partial \rho^{f}} \mathbf{I} \\
\boldsymbol{\pi}_{m^{\prime}}=\boldsymbol{\pi}_{m}-p \frac{\partial \phi}{\partial \mathbf{F}^{s}} \operatorname{grad} \mathbf{F}^{s}
\end{gathered}
$$

where $\left(\mathbf{T}_{m}^{\alpha}, \boldsymbol{\pi}_{m}\right)$ are evaluated on $\mathcal{S}$ and $p$ is arbitrary.

The constraint of intrinsic incompressibility first proposed by Mills [10] as

$$
\frac{\rho^{s}}{\rho^{s T}}+\frac{\rho^{f}}{\rho^{f T}}=1
$$

is derived assuming that the mixture is saturated and that the true densities, $\rho^{s T}$ and $\rho^{f T}$, are constant. Writing Eq. (13) in the form Eq. (11), invoking Eq. (12), and recalling Eqs. (6)-(9), the final constitutive equations for the partial stresses and the momentum supply in our incompressible special theory become (dropping the subscripts)

$$
\begin{gathered}
\mathbf{T}^{s}=-\phi^{s} p \mathbf{I}+\rho^{s} \frac{\partial \psi^{s}}{\partial \mathbf{F}^{s}} \mathbf{F}^{s^{T}} \equiv-\phi^{s} p \mathbf{I}+\hat{\mathbf{T}}^{s} \\
\mathbf{T}^{f}=-\phi^{f} p \mathbf{I}-\rho^{f^{2}} \frac{\partial \psi^{f}}{\partial \rho^{f}} \mathbf{I} \equiv-\phi^{f} p \mathbf{I}+\hat{\mathbf{T}}^{f} \\
\boldsymbol{\pi}=-\frac{\operatorname{grad} \rho^{s}}{\rho^{s T}} p+{ }_{e} \boldsymbol{\pi} \mathbf{a}
\end{gathered}
$$

where we have introduced the volume fractions $\phi^{\alpha}=\rho^{\alpha} / \rho^{\alpha} T$. These constitutive equations are less restrictive than their counterpart (Eqs. (30)) of the special theory of Kwan et al. [12] by the inclusion of the determinate term in the partial fluid stress Eq. (15).

It is worthwhile to note that our definition for a constrained mixture resolves any apparent contradictions between the restrictions Eq. (5), and the constraint Eq. (11). In particular, we define Eq. (5) $)_{1}$ as the partial derivative of the solid free energy function, $\psi^{s}$, with respect to the fluid density, $\rho^{f}$, while holding $\mathbf{F}^{s}$ (and thus $J^{S}=\operatorname{det} \mathbf{F}^{s}$ ) fixed. If $\psi^{s}$ was only defined on $\mathcal{S}$, then this calculation would not be possible, because $\psi^{s}$ could not be calculated at different values of $\rho^{f}$ while holding $J^{s}$ fixed. In our definition for a constrained mixture, $\psi^{s}$ is inherited from an unconstrained mixture and, consequently, is defined off of $\mathcal{S}$ in the 
10-dimensional space $\left(\mathbf{F}^{s}, \rho^{f}\right)$. In other words, our development ensures that the restrictions, Eq. (5), are satisfied since $\psi^{s}$ can be calculated at different values of $\rho^{f}$ while holding $J^{s}$ fixed, even though the condition, Eq. (11), ultimately allows one to express $\rho^{f}$ in terms of $J^{s}$. Thus, the geometric treatment of internally constrained mixtures appears to be the first to resolve any apparent contradictions between the constitutive restrictions obtained by Atkin and Craine [26] and the constraint of intrinsic incompressibility proposed by Mills [10].

\section{Analysis}

In confined compression, the specimen is inserted into a rigid cylindrical confining chamber, which prevents radial expansion, and a constant compressive displacement rate is applied during the ramp phase by a rigid platen $\left(X_{3}=0\right.$; Fig. 1$)$. It is convenient to define a reference frame relative to the moving rigid platen; this definition suggests boundary conditions of zero solid and fluid displacements at $X_{3}=0$ during the entire test. The specimen is supported at its other end by a free-draining porous platen $\left(X_{3}\right.$ $=h)$. This suggests a prescribed solid displacement boundary condition and a zero fluid pressure boundary condition at $X_{3}=h$ during the ramp phase. During the relaxation phase, the rigid platen is held at a fixed displacement, suggesting a fixed solid displacement boundary condition and zero fluid pressure boundary condition $\left(X_{3}=h\right)$ during the relaxation phase. The deformation of both constituents is assumed to be one-dimensional. Thus, the boundary conditions for the stress relaxation protocol are:

$$
\begin{gathered}
t<t_{0}: U(0, t)=v_{3}^{f}(0, t)=0 ; \quad U(h, t)=-\dot{U} t ; T_{33}^{f}(h, t)=0 \\
t_{0} \leqslant t \leqslant t_{f}: \quad U(0, t)=v_{3}^{f}(0, t)=0 ; \quad U(h, t)=-U t_{0} ; \\
T_{33}^{f}(h, t)=0
\end{gathered}
$$

where $U$ is the solid displacement, $t_{0}$ is the duration of the ramp phase, and $t_{f}-t_{0}$ is the duration of the relaxation phase. The assumed initial conditions are

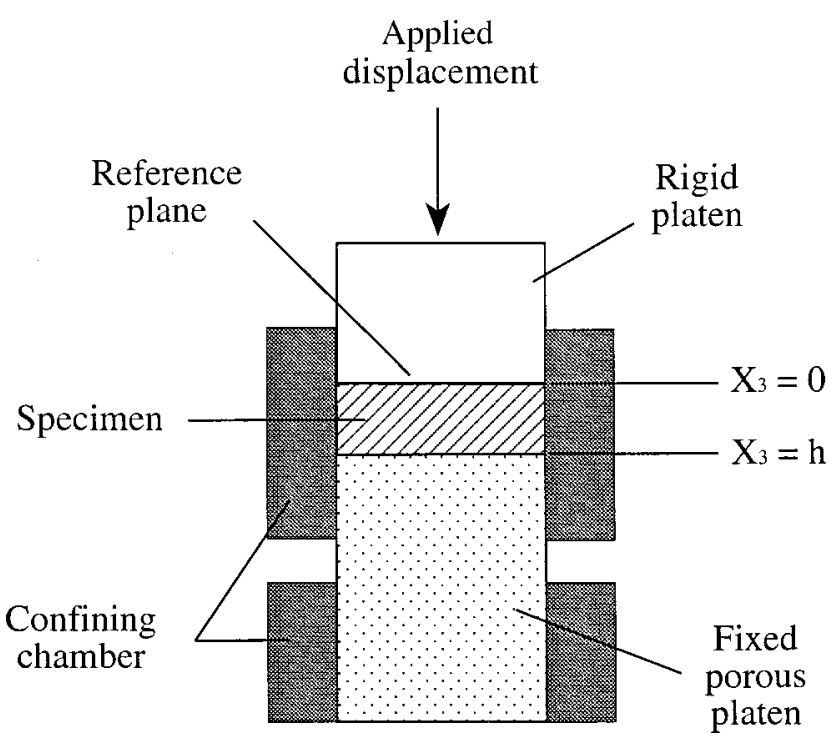

Fig. 1 Schematic of experimental design. The specimen is supported between a rigid platen at $X_{3}=0$, a fixed porous platen at $X_{3}=h$, and a rigid confining chamber on its periphery. The displacement is applied to the rigid platen. The junction between the rigid platen and the specimen (i.e., $X_{3}=0$ ) is chosen as a "stationary" reference plane. Thus, the displacements are zero at $X_{3}=0$ and are prescribed at $X_{3}=h$.

$$
U\left(X_{3}, 0\right)=v_{3}^{f}\left(X_{3}, 0\right)=T_{33}^{s}\left(X_{3}, 0\right)=T_{33}^{f}\left(X_{3}, 0\right)=0
$$

The one-dimensional solid deformation is $x_{3}\left(X_{3}, t\right)=X_{3}$ $+U\left(X_{3}, t\right)$ where $X_{3}$ is the reference position of the solid particle. The solid stretch is given by $\lambda_{3}\left(X_{3}, t\right)=1$ $+\partial U\left(X_{3}, t\right) / \partial X_{3}$.

An important relation to obtain from our special theory is that of the equilibrium stress of the solid phase, which is obtained by setting the fluid stress to zero in Eq. (15), since it is assumed that the fluid stress is zero at all points in the specimen at equilibrium. We then obtain from Eqs. (14)-(15) the result

$$
T_{33, \mathrm{eq}}^{s}=\hat{T}_{33}^{s}-\frac{\phi^{s}}{\phi^{f}} \hat{T}_{33}^{f} \text {, at equilibrium. }
$$

The result, Eq. (19), also represents the surface stress due to the fluid pressure boundary condition at $X_{3}=h$. We define the determinate part of the momentum supply as

$$
{ }_{e} \pi_{33}\left(v_{3}^{s}-v_{3}^{f}\right)=\frac{\phi^{f^{2}}}{k}\left(v_{3}^{s}-v_{3}^{f}\right)
$$

where $k$ is the nonlinear permeability function. The governing partial differential equation for the solid displacement is

$$
\frac{k}{1+\frac{\partial U}{\partial X_{3}}}\left(\frac{\partial \hat{T}_{33}^{s}}{\partial \lambda_{3}}-\frac{\phi_{0}^{s}}{1+\frac{\partial U}{\partial X_{3}}-\phi_{0}^{s}} \frac{\partial \hat{T}_{33}^{f}}{\partial \lambda_{3}}\right) \frac{\partial^{2} U}{\partial X_{3}^{2}}=\frac{\partial U}{\partial t} .
$$

By setting the determinate part of the fluid stress to zero in Eq. (21), we obtain the governing equation used by other authors $[14,22]$.

We assume isotropy and use the constitutive equation for the solid free energy function proposed by Holmes and Mow [21]

$$
\rho_{0}^{s} \psi^{s}=\frac{\alpha_{0}}{I_{3}^{\beta}} \exp \left[\alpha_{1}\left(I_{1}-3\right)+\alpha_{2}\left(I_{2}-3\right)\right]
$$

where $\alpha_{i}, \beta$ are material constants and $I_{i}$ is the $i$ th invariant of $\mathbf{C}^{s}$. The constant $\alpha_{0}$ has dimensions of stress while the remaining constants are dimensionless. From Eq. (14) we can calculate the normal solid stress component in the loading direction as

$$
\hat{T}_{33}^{s}=\frac{2 \alpha_{0}}{\lambda_{3}^{2 \beta-1}}\left(\alpha_{1}+2 \alpha_{2}-\frac{\beta}{\lambda_{3}^{2}}\right) \exp \left[\left(\alpha_{1}+2 \alpha_{2}\right)\left(\lambda_{3}^{2}-1\right)\right] .
$$

We specify the fluid free energy function based on an equation used by Shi et al. [30]

$$
\psi^{f}=-\frac{Q}{\rho^{f T}}\left[\ln \left(1-\frac{\rho^{f}}{\rho^{f T}}\right)\right]
$$

where $Q>0$ is a material constant with dimensions of stress. In writing Eq. (24), we have scaled the material constant $Q$ and the fluid density $\rho^{f}$ by dividing by the constant $\rho^{f T}$ for matters of convenience. We obtain from Eqs. (13), (15), and (24), and the continuity equations, the determinate fluid stress

$$
\hat{T}_{33}^{f}=-\frac{Q}{\phi_{0}^{s}} \frac{\left(\lambda_{3}-\phi_{0}^{s}\right)^{2}}{\lambda_{3}} .
$$

To arrive at Eq. (25), we first calculate $\partial \psi^{f} / \partial \rho^{f}$ in terms of $\rho^{f}$ and then evaluate this quantity on the constraint manifold, allowing us to rewrite the results in terms of $\lambda_{3}$ (this procedure is consistent with our definition of a constrained mixture).

The primary reasons that we chose the natural logarithm function for $\psi^{f}$ are that it allows us to introduce a nonlinear function while only introducing one additional material constant, and that it $\left(\psi^{f}\right)$ and the determinate fluid stress appear to be well-behaved. In proposing $\psi^{s}$, Holmes and Mow [21] introduced the plausible restriction that $\psi^{s}$ becomes unbounded as $J^{s} \rightarrow \infty$. In the present 
application, as $\phi^{f} \rightarrow 1, \phi^{s} \rightarrow 0 \Rightarrow \rho^{s} \rightarrow 0 \Rightarrow J^{s} \rightarrow \infty$. It seems physically reasonable that both free energy functions should prevent the Jacobian from becoming unbounded; thus, one reason we chose the natural logarithm is that $\psi^{f}$ becomes unbounded as $\phi^{f} \rightarrow 1$. Another reason for choosing the natural logarithm is that $\psi^{f}$ approaches zero as $\phi^{f} \rightarrow 0$, which again seems physically reasonable, and which provides a vanishing determinate fluid stress as $\phi^{f} \rightarrow 0$.

By assuming that there exist a stress-free reference configuration, we obtain from Eqs. (19), (23), and (25) the restriction

$$
\alpha_{1}+2 \alpha_{2}=\frac{-Q\left(1-\phi_{0}^{s}\right)}{2 \alpha_{0}}+\beta .
$$

For the confined compression deformation, we have to determine three unknown material constants representing the solid and fluid stresses: $\alpha_{0}, \beta$, and $Q$. The final constitutive equation that we use is the one-dimensional nonlinear permeability function $k$ proposed by Holmes and Mow [21]

$$
k=k_{0}\left(\frac{\lambda_{3}-\phi_{0}^{s}}{1-\phi_{0}^{s}}\right) \exp \left[M\left(\lambda_{3}^{2}-1\right) / 2\right]
$$

where $k_{0}$ and $M$ are material constants. The governing partial differential Eq. (21) can be written in terms of $U$ and its partial derivatives and the material constants. For comparison with other studies in the biomechanics literature, it is useful to compute the aggregate modulus $H_{A 0}$ defined as [21]:

$$
\begin{aligned}
H_{A 0}=\left.\frac{\partial T_{33, \mathrm{eq}}^{s}}{\partial \lambda_{3}}\right|_{\lambda_{3}=1}= & Q\left(1-\phi_{0}^{s}\right)\left(\frac{Q\left(1-\phi_{0}^{s}\right)}{2 \alpha_{0}}+\beta-1\right)+4 \beta \alpha_{0} \\
& +Q \phi_{0}^{s} .
\end{aligned}
$$

\section{Experimental Protocol}

Thirty-two annulus fibrosus specimens were harvested from the middle-lateral portion of intact intervertebral discs (IVD's) from the $L 23, L 34$, and $L 45$ motion segments of seven intact human spines (Table 1). The motion segments were removed by sawing through the mid-transverse plane of the adjacent vertebral bodies and cut in half along the mid-sagittal plane using a precision diamond saw (Exakt 3000, Electromechanical Cutter, Norderstedt, Germany). A degeneration grade was assigned according to the Thompson scale [31]; only grade I or II specimens were used. A block of the IVD was removed from the lateral portion and cut into two pieces, one for obtaining axial loading slices and one for obtaining radial loading slices (Fig. 2). These tissue blocks were wrapped in gauze soaked in physiological saline $(0.15 \mathrm{M} \mathrm{NaCl}$ solution), placed in a sealed bag and stored at $-20^{\circ} \mathrm{C}$. After 24 hours, the tissue blocks were removed from the freezer and 2-mmthick slices were cut on a vibratome (MicroCut H1200, Energy Beam Sciences, Agawam, MA) while spraying with tissue freezing spray (Quick-Freeze, Electron Microscopy Sciences, Fort Washington, PA) to keep the tissue block from thawing. Each slice was wrapped in gauze soaked in physiological saline and stored at $-20^{\circ} \mathrm{C}$ until testing.

A servo-hydraulics materials testing machine (MTS Bionix 858, Material Test System, Eden Prairie, MN) was used to perform the experiments. Displacement was measured with the testing system LVDT and force was measured with a 1000 gram load cell (Model 31, Sensotec, Columbus, OH). Without the specimen in place, the load cell was zeroed and the MTS crosshead was lowered until the load cell registered a negative reading at contact. This determined the initial height (IH). The internal confining chamber was removed and used to punch a cylindrical test specimen from the 2-mm-thick tissue slice (diameter $=5 \mathrm{~mm}$ ). The internal confining chamber was replaced and the final height $(\mathrm{FH})$ was determined after lowering the crosshead until the load cell registered a negative reading. The tissue thickness $(h)$ was calcu-
Table 1 Level, degeneration grade, age, and sex for all intervertebral discs harvested for experimentation. Axial $(A)$ and radial $(R)$ loading test specimens (one each) were obtained from each disc (an axial specimen is one with the axial plane cut normal to the loading direction).

\begin{tabular}{ccccc}
\hline Disc \# & Level & Disc Grade & Age & Sex \\
\hline I & L23 & II & 28 & M \\
2 & L34 & II & 28 & M \\
3 & L45 & II & 28 & M \\
4 & L23 & II & 36 & M \\
5 & L34 & II & 36 & M \\
6 & L23 & I & 40 & F \\
7 & L34 & I & 40 & F \\
8 & L45 & II & 40 & F \\
9 & L23 & I & 40 & M \\
10 & L45 & II & 40 & M \\
11 & L34 & I & 41 & F \\
12 & L23 & II & 40 & M \\
13 & L34 & I & 40 & M \\
14 & L45 & II & 40 & M \\
15 & L23 & I & 40 & M \\
16 & L34 & I & 40 & M \\
\hline
\end{tabular}

lated as $h=\mathrm{FH}-\mathrm{IH}$. Next, a tare cycle loaded the specimen to 30 gf and allowed relaxation for $2000 \mathrm{~s}$. The tare cycle was followed by four stress-relaxation cycles each with a $2000 \mathrm{~s}$ ramp phase conducted at a fixed displacement rate of $0.0001 \mathrm{~mm} / \mathrm{s}$ (corresponding to a total displacement of $0.2 \mathrm{~mm}$ ) followed by a $6000 \mathrm{~s}$ relaxation phase. The data collected were the solid surface stress and displacement $\left(X_{3}=h\right)$ in the loading direction. Upon completion of the experiment, the test specimen was removed from the confining chamber, gently blotted to remove excess water, and placed in a vial. The specimen and vial was weighed (WT1) and then heat-dried at $65^{\circ} \mathrm{C}$ for 24 hours. The specimen and vial were weighed again (WT2), the dried specimen was removed from the vial, and the vial alone was weighed (WT3). Initial water content was then calculated as $\phi_{0}^{f}=(\mathrm{WT} 1-\mathrm{WT} 2+$ w.p.o. $) /$ (WT1-WT3+w.p.o.) where w.p.o.= water pushed out during experiment.
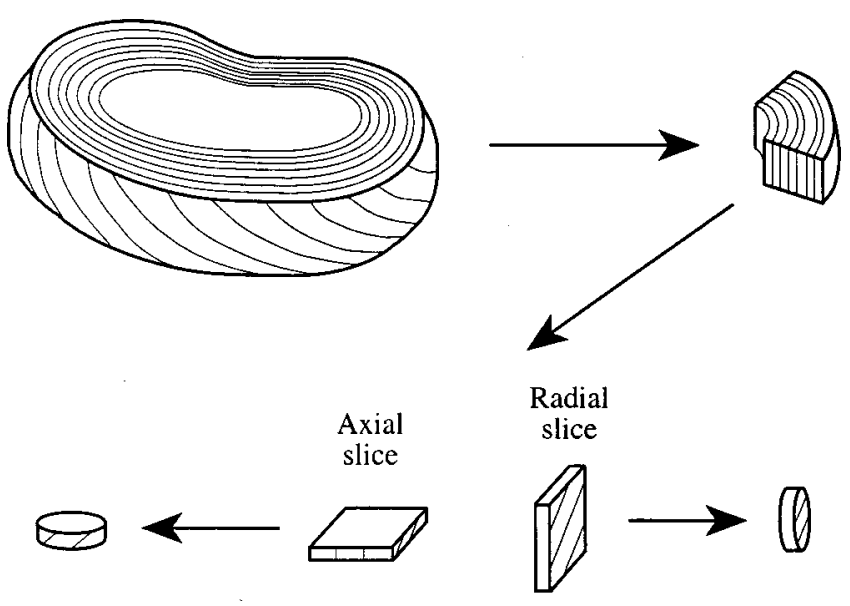

Fig. 2 Protocol for obtaining cylindrical axial and radial test specimens from an intact intervertebral disc. A lateral block of the disc was removed and 2-mm-thick axial and radial slices were cut from the block. A cylindrical test specimen was punched from each slice using the internal confining chamber. 


\section{Solution Procedure}

The offset stress was defined as the stress at the end of the tare step (i.e., $2000 \mathrm{~s}$ after application of the tare load). This offset stress was subtracted from the remaining data. The equilibrium stress was identified as that corresponding to the final value of stress in each loading cycle (i.e., at intervals of 8000 s). The equilibrium stretch was calculated by subtracting from unity the total applied displacement (i.e., either $0.2,0.4,0.6$, or $0.8 \mathrm{~mm}$ ) divided by the initial tissue height. A nonlinear fitting algorithm in DeltaGraph (DeltaPoint, Monterey, CA) was used to fit the theoretical equilibrium stress, Eq. (19), using Eqs. (23), (25), and (26) to determine the material constants $\alpha_{0}, \beta$, and $Q$. This curve-fit was first performed with the material constant $Q=0$ corresponding to the theory used by other authors to obtain $\alpha_{0}$ and $\beta$. The curve-fit was repeated with $Q$ as a variable to obtain $\alpha_{0}, \beta$, and $Q$.

Then, initial values of the permeability constants $k_{0}$ and $M$ were assumed and the one-dimensional partial differential Eq. (21) subject to the boundary conditions, Eq. (17), and initial conditions, Eq. (18), was solved numerically for the solid displacement $U\left(X_{3}, t\right)$. The numerical solution was achieved using the 1987 version of the program PDECOL [32]. The solid stress at $X_{3}=h$ was calculated and used in a custom optimization scheme using the simplex method to determine the values of the permeability constants $k_{0}$ and $M$ that maximized the $R^{2}$ correlation statistic between our predicted solid stress at $X_{3}=h$ and the experimental data. The optimization scheme requires as input the initial values of the permeability constants $k_{0}$ and $M$, the lengths of the simplex triangle sides $\Delta k_{0}$ and $\Delta M$, and the maximum number of iterations. A numerical solution is first obtained for three points in the two-dimensional $\left(k_{0}, M\right)$ space corresponding to the vertices of the triangle: $\left(k_{0}, M\right),\left(k_{0}+\Delta k_{0}, M\right)$, and $\left(k_{0}, M+\Delta M\right)$. For each of the vertices of the simplex, the $R^{2}$ correlation statistic between our predicted solid stress at $X_{3}=h$ and the experimental data is calculated. The vertex with the least $R^{2}$ value is rejected and a new vertex is defined; if the vertex with the least $R^{2}$ value corresponds to the most recently created vertex, then the vertex with the lower $R^{2}$ value of the remaining two is rejected. The optimization procedure continues in this fashion until the maximum number of iterations is achieved. The optimization procedure was performed with both sets of material constants $\alpha_{0}, \beta$, and $Q$ representing both the present theory and that used by other authors.

All statistical analyses were performed using the SYSTAT statistical software package (V5.2, SYSTAT, Inc., Evanston IL). Normal probability (rankit) plots were first generated for each outcome variable $\left(\alpha_{0}, \beta, Q, H_{A 0}, k_{0}, M\right)$ to determine whether these data followed an underlying normal distribution. All variables except $M$ were positively skewed. Therefore, in order to test whether the categorical variables (employed theory or specimen orientation) significantly influenced the parameters of interest $\left(\alpha_{0}, \beta, Q, H_{A 0}, k_{0}, M\right)$ we utilized a paired $t$-test, with the null hypothesis that the paired difference, e.g., $\Delta \alpha_{0}$, was equal to zero. In this case we demonstrated that the paired differences for all dependent variables followed a normal distribution.

\section{Results}

The specimens exhibited a typical stress-relaxation response to the applied loading conditions (Fig. 3). Both the equilibrium solid stress versus stretch (Fig. 4) and the solid surface stress time history (Fig. 5) responses were well described by the present theory. For all specimens, the initial water content was $\phi_{0}^{f}$ $=0.84 \pm 0.03$, the initial specimen thickness was $h=2.42$ $\pm 0.43 \mathrm{~mm}$, and the maximum applied stretch was $\lambda_{\max }=0.66$ \pm 0.05 . No swelling was observed in the tare step and the offset stress for all specimens was $-0.005 \pm 0.004 \mathrm{MPa}$.

Table 2 lists the results (mean \pm 1 standard deviation) for material constants determined using the theory proposed in this paper and that of Holmes and Mow [21] $(n=32)$ and for axial and radial specimens $(n=16)$. Paired $t$-tests revealed a significant difference due to orientation in the correlation coefficient for the transient data curvefit (Table 2). The values of $\Delta H_{A 0}(p$ $<0.0001), \Delta k_{0}(p<0.000001)$, and $\Delta \beta(p<0.001)$ due to the choice of theory were significantly different from zero, while $\Delta M(p=0.053)$ was a borderline case (Table 3$)$. No significant differences in orientation existed for these parameters.

The aggregate modulus $H_{A 0}$ and the initial permeability constant $k_{0}$ were the only material constants significantly correlated with initial water content $\phi_{0}^{f}(p<0.001)$ (Figs. 6 and 7). Nonlinear power law relationships were fit to the data to obtain

$$
\begin{gathered}
H_{A 0}=0.008\left(\phi_{0}^{f}\right)^{-13.1} \mathrm{MPa} \\
k_{0}=0.1156\left(\phi_{0}^{f}\right)^{15.41} \mathrm{~mm}^{4} / \mathrm{N}-\mathrm{s}
\end{gathered}
$$

resulting in $R^{2}$ values of 0.49 and 0.38 , respectively.

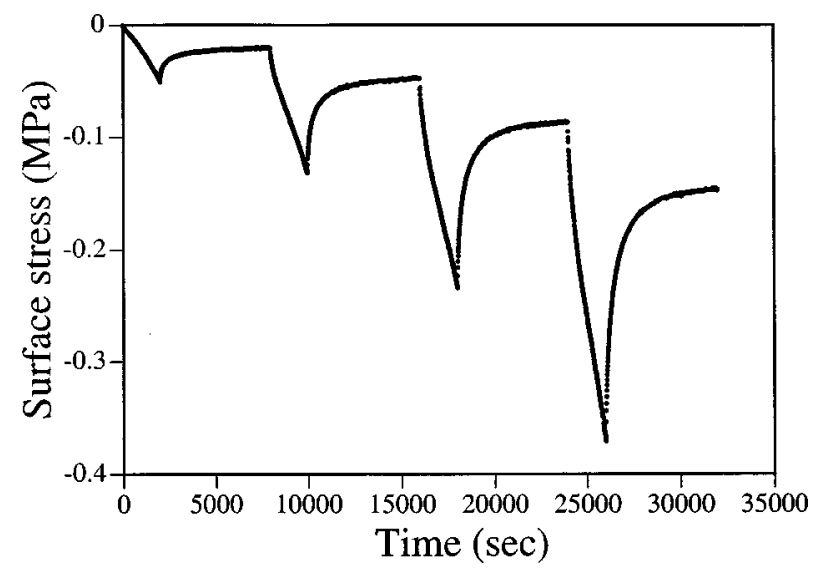

Fig. 3 Experimental data measured for the solid stress at $X_{3}$ $=h$ (surface stress) versus time for four consecutive stressrelaxation cycles during test $1 \mathrm{~A}$.

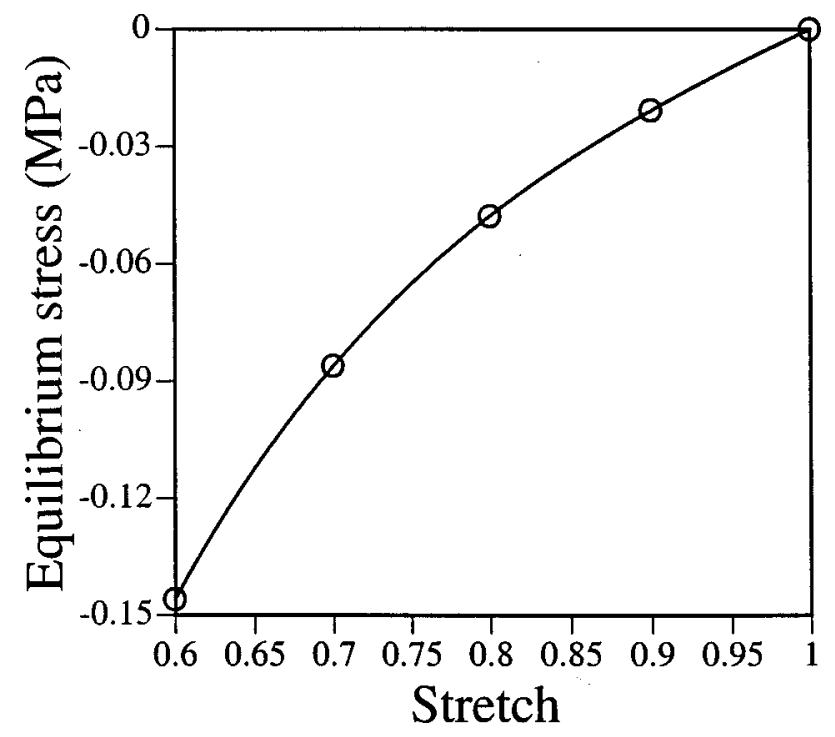

Fig. 4 Theoretical curve-fit using Eqs. (19), (23), (25), (26) to the experimental data representing the equilibrium solid stress versus the measured equilibrium stretch $\lambda_{3}$ for test $1 \mathrm{~A}$. Circles $=$ experimental points, line $=$ theoretical fit. The material constants were $\alpha_{0}=0.074 \mathrm{MPa}, \beta=0.658$, and $Q=0.027 \mathrm{MPa}$, and the $R^{2}$ value was 1.000 . With $Q=0$, the $R^{2}$ value was 1.000 (not shown). 


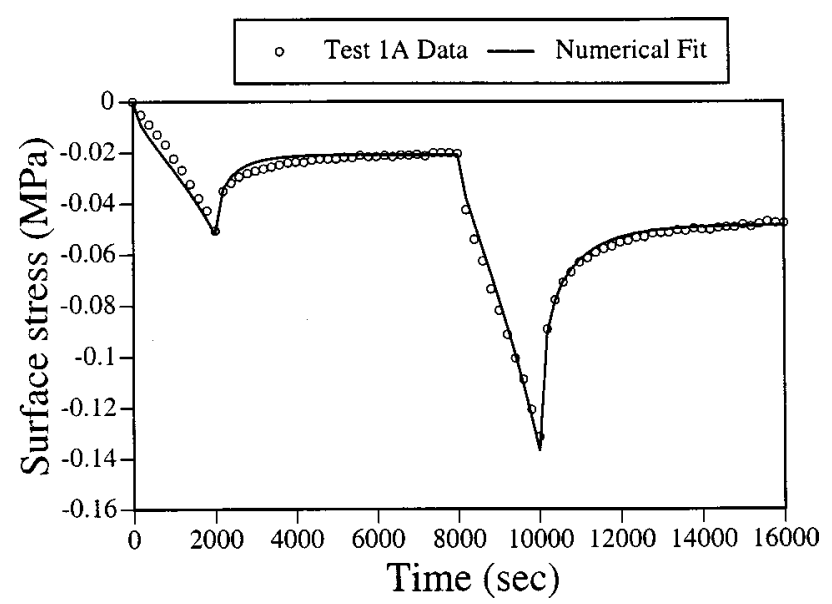

Fig. 5 Optimized numerical solution to Eq. (21) to achieve a best-fit regression to the experimental data measured for the solid stress at $X_{3}=h$ (surface stress) versus time for the first two stress-relaxation cycles for test $1 \mathrm{~A}$. Circles=experimental points, line $=$ theoretical fit. The material constants were $k_{0}$ $=0.0061 \mathrm{~mm}^{4} / \mathrm{N}-\mathrm{s}$ and $M=9.5$ and the $R^{2}$ value was 0.989 . With $Q=0$, the $R^{2}$ value was 0.991 (not shown).

Table 2 Results (mean \pm 1 standard deviation) for material constants determined using the theory proposed in this paper and that of Holmes and Mow [21] $(n=32)$ and for axial and radial specimens $(n=16) . \alpha_{0}, Q, H_{A 0}$ are in $\mathrm{MPa}, k_{0}$ is in $\mathrm{mm}^{4} / \mathrm{N}$-s. $R^{2 *}$ is the correlation statistic for the equilibrium stress versus stretch fit; $R^{2^{* *}}$ is the correlation statistic for the surface stress history fit. Paired $t$-tests revealed no significant differences in $R^{2 *}$ and $R^{2 * *}$ due to the choice of theory; a significant difference existed in $R^{2 * *}$ due to orientation ( $p$ $<0.05)$.

Theory

Orientation

\begin{tabular}{lllll}
\hline & $\begin{array}{l}\text { Present } \\
\text { theory }\end{array}$ & $\begin{array}{l}\text { Holmes and } \\
\text { Mow(1990) }\end{array}$ & Axial & Radial \\
\hline$\alpha_{0}$ & $0.110 \pm 0.138$ & $0.107 \pm 0.180$ & $0.116 \pm 0.127$ & $0.104 \pm 0.153$ \\
$\beta$ & $0.606 \pm 0.563$ & $1.183 \pm 1.378$ & $0.507 \pm 0.403$ & $0.706 \pm 0.686$ \\
$\mathrm{Q}$ & $0.077 \pm 0.084$ & $\mathrm{~N} / \mathrm{A}$ & $0.077 \pm 0.084$ & $0.077 \pm 0.076$ \\
$\mathrm{H}_{\mathrm{A0}}$ & $0.116 \pm 0.127$ & $0.138 \pm 0.083$ & $0.123 \pm 0.070$ & $0.095 \pm 0.072$ \\
$\mathrm{R}^{2 *}$ & $0.994 \pm 0.009$ & $0.993 \pm 0.009$ & $0.998 \pm 0.002$ & $0.990 \pm 0.011$ \\
$\mathrm{k}_{0}$ & $0.013 \pm 0.015$ & $0.016 \pm 0.016$ & $0.011 \pm 0.014$ & $0.014 \pm 0.017$ \\
$\mathrm{M}$ & $9.091 \pm 2.049$ & $9.309 \pm 1.758$ & $8.700 \pm 1.388$ & $9.494 \pm 2.533$ \\
$\mathrm{R}^{2 * *}$ & $0.951 \pm 0.059$ & $0.956 \pm 0.026$ & $0.963 \pm 0.018$ & $0.940 \pm 0.068$
\end{tabular}

\section{Discussion}

We derived a special theory of an intrinsically incompressible mixture, which was applied to stress-relaxation data from annulus fibrosus specimens. The values for the initial water content $\phi_{0}^{f}$, the initial elastic modulus $H_{A 0}$, and the permeability constants $k_{0}$ and $M$ are substantially different from those obtained for other confined compression studies with human annulus fibrosus $[13,14]$. These discrepancies can be explained by the choice of reference configuration chosen for the experimental studies. In the current study, care was taken to keep the specimens well hydrated at all steps of the specimen preparation process and the value of initial water content $\phi_{0}^{f}=0.84 \pm 0.03$ is substantially higher than that reported by Best et al. [13] of $\phi_{0}^{f}=0.70 \pm 0.05$. As a consequence of a lower water content, in these previous experiments,
Table 3 Results (mean \pm 1 standard deviation) for paired differences in material constants determined using the theory proposed in this paper and that of Holmes and Mow [21] $(n$ $=32$ ) and for axial and radial specimens $(n=16) . \Delta \alpha_{0}$ $=\left(\alpha_{0}\right)_{H M}-\left(\alpha_{0}\right)_{K L}$ or $\Delta \alpha_{0}=\left(\alpha_{0}\right)_{A X}-\left(\alpha_{0}\right)_{\mathrm{RAD}}$ where $\left(\alpha_{0}\right)_{H M}$ is obtained using Holmes and Mow [21], $\left(\alpha_{0}\right)_{K L}$ is obtained using the theory proposed in this paper, $\left(\alpha_{0}\right)_{A X}$ is for axial specimens (using the present theory), and ( $\left.\alpha_{0}\right)_{\mathrm{RAD}}$ is for radial specimens (using the present theory). $\Delta \beta, \Delta Q, \Delta H_{A 0}, \Delta k_{0}$, and $\Delta M$ are defined in a similar fashion. Paired $t$-tests were used to investigate whether the paired differences were significantly different than zero. The values of $\Delta H_{A 0}(p<0.0001), \Delta k_{0}(p$ $<0.000001)$, and $\Delta \beta \quad(p<0.001)$ due to the choice of theory were significantly different than zero, while $\Delta M$ was a borderline case $(p=0.053)$. No significant differences in orientation existed.

\begin{tabular}{lll}
\hline & Theory & Orientation \\
\hline$\Delta \alpha_{0}$ & $-0.003 \pm 0.119$ & $0.012 \pm 0.149$ \\
$\Delta \beta$ & $0.576 \pm 0.879$ & $-0.199 \pm 0.678$ \\
$\Delta \mathrm{Q}$ & $\mathrm{N} / \mathrm{A}$ & $0.001 \pm 0.125$ \\
$\Delta \mathrm{H}_{\mathrm{A} 0}$ & $0.029 \pm 0.033$ & $0.028 \pm 0.082$ \\
$\Delta \mathrm{k}_{0}$ & $0.004 \pm 0.003$ & $-0.002 \pm 0.012$ \\
$\Delta \mathrm{M}$ & $0.272 \pm 0.765$ & $-0.675 \pm 1.954$ \\
\hline
\end{tabular}

the specimens generated high swelling pressures during the tare step of approximately $-0.12 \pm 0.06 \mathrm{MPa}$, which was subsequently defined as an offset stress and subtracted off of the remaining data. In contrast, none of our specimens exhibited noticeable swelling and our offset stress of $-0.005 \pm 0.004 \mathrm{MPa}$ is two orders of magnitude lower. As a result, our reference configuration is closer to that of a stress-free state for the mixture, which is important since a restriction on the elasticity constants (e.g., see Eq. (26)) is developed from assuming the existence of a stressfree configuration. If a reference configuration with a nonvanishing stress is chosen, then considerable errors may be introduced.

As a consequence of our chosen reference configuration, we arrived at different material constants than those presented by others. In the present study, we obtained values of $H_{A 0}=0.116$

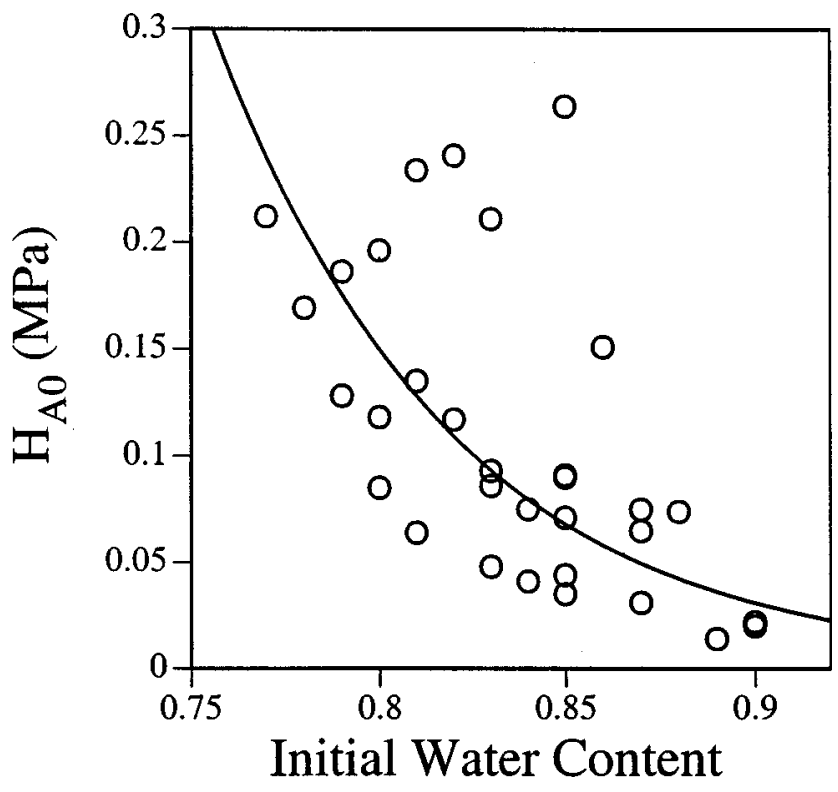

Fig. 6 Plot of equilibrium aggregate modulus $H_{A 0}$ versus initial water content $\phi_{0}^{f}$. A nonlinear power law relationship $H_{A 0}$ $=0.008\left(\phi_{0}^{f}\right)^{-13.1} \mathrm{MPa}$ best describes the curve, $R^{2}=0.49$. This dependence was significant $(p<0.001)$. 


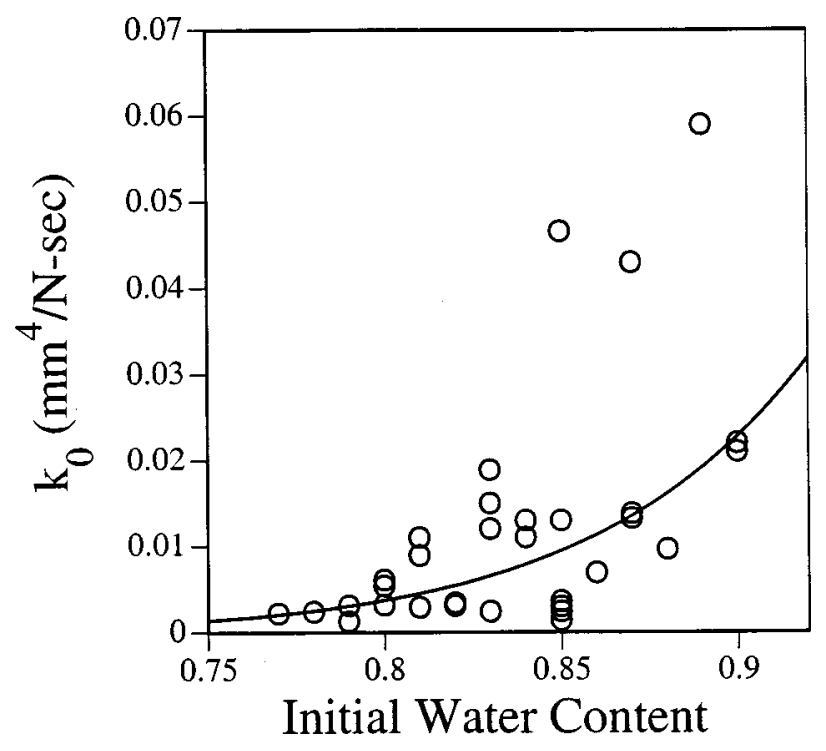

Fig. 7 Plot of initial permeability constant $k_{0}$ versus initial water content $\phi_{0}^{f}$. A nonlinear power law relationship $k_{0}$ $=0.1156\left(\phi_{0}^{f}\right)^{15.41} \mathrm{~mm}^{4} / \mathrm{N}$-s best describes the curve, $R^{2}=0.38$. This dependence was significant $(p<0.001)$.

$\pm 0.127 \mathrm{MPa}, \quad k_{0}=0.013 \pm 0.015 \mathrm{~mm}^{4} / \mathrm{N}-\mathrm{s}, \quad$ and $M=9.09 \pm 2.05$ whereas Best et al. [13] reported values of $H_{A 0}=0.38$ $\pm 0.16 \mathrm{MPa}, k_{0}=0.00025 \pm 0.00011 \mathrm{~mm}^{4} / \mathrm{N}-\mathrm{s}$, and Iatridis et al. [14] reported values of $H_{A 0}=0.56 \pm 0.21 \mathrm{MPa}, k_{0}=0.00018$ $\pm 0.00007 \mathrm{~mm}^{4} / \mathrm{N}-\mathrm{s}$, and $M=1.5 \pm 1.6$. These differences are all physically intuitive. With a higher water content, the equilibrium elastic modulus should be lower, as there exists less solid matrix per unit mixture area to support the compressive load while the fluid is assumed to experience zero stress at equilibrium. Also, with a higher water content, there exists less frictional resistance to fluid flow since the solid is less compressed and its pores are larger, and thus the permeability should be higher. As our results for the initial permeability constant are two orders of magnitude higher than those reported with a lower initial water content, the material constant $M$ representing the degree of nonlinearity in the exponential permeability function should be higher. In fact, to obtain a water content of 0.7 after starting with our initial water content $\phi_{0}^{f}=0.84$, a stretch of 0.53 must be applied during the experiment. Using $\lambda_{3}=0.53$ and our mean permeability constants of $k_{0}=0.013 \mathrm{~mm}^{4} / \mathrm{N}$-s and $M=9.09$, the permeability $k$ at $\lambda_{3}$ $=0.53$ was calculated to be $k=0.00021 \mathrm{~mm}^{4} / \mathrm{N}-\mathrm{s}$, which lies between the mean values reported by Best et al. [13] and Iatridis et al. [14].

The material properties did not depend on orientation. This result can be expected as the highly anisotropic response of the annulus is primarily due to the presence of collagen fibers, which offer little resistance in compression. Our results suggest that the ground substance may be isotropic and that an appropriate anisotropic formulation for the annulus may be obtained by using a fiber-reinforced theory in which the annulus is modeled as an isotropic ground substance reinforced with families of fibers [33]. There did exist significant differences in the values obtained for $H_{A 0}$ and $k_{0}$ depending on the choice of the theory that was used (Table 3). Although these results are statistically significant, there is some doubt as to the significance from a biological or a clinical perspective since the variabilities due to biological differences are considerably more important than those due to the choice of theory. Yet, the biological importance of these differences needs to be evaluated in the context of future studies that use these data to address biological questions. Regardless, the present study is the first to quantify the effect of constitutive assumptions that have been imposed yet never directly justified in previous experimental and theoretical studies in the biomechanics literature.

A possible limitation is that there exists some uncertainty as to the plausibility of the boundary conditions, Eq. (17), in an experimental protocol employing a free-draining porous platen. In particular, Buschmann et al. [34] have shown that a more precise specification of the boundary conditions between the specimen and both the porous platen and the confining chamber results in substantially different material constants as determined from confined compression experimental data. We invoked the boundary conditions, Eq. (17), and initial conditions, Eq. (18), that are the same as those used by other authors in the biomechanics literature $[21,14]$ for a confined compression stress-relaxation protocol. For a creep protocol in which a prescribed load is applied at $X_{3}=h$, it is usually assumed that the solid matrix supports all of the load at $X_{3}=h[8]$. Thus, Eq. (17) would be changed by prescribing a solid stress, as opposed to a solid displacement, boundary condition at $X_{3}=h$. It should be pointed out that there exists considerable uncertainty regarding the specification of boundary conditions in the mixture theory literature. In particular, Rajagopal and Tao [35] have discussed two methods for specifying traction boundary conditions: a method of "splitting" the total traction on the boundaries using the volume fractions of the constituents, and a method invoking a saturation condition on the boundary. In addition, Reynolds and Humphrey [36] have proposed a velocity boundary condition for a solid-fluid mixture.

Another possible limitation of the present study is that we have produced a constrained fit to the experimental data because the values of the elasticity constants $\left(\alpha_{0}, \beta, Q\right)$ affect the optimized values of the permeability constants $\left(k_{0}, M\right)$, although the former parameters are fixed during the optimization. However, we believe that the physics of the problem suggest that the best way to fit the transient data is the method that we have chosen. In particular, we have determined the three constants $\left(\alpha_{0}, \beta, Q\right)$ by fitting the equilibrium stress versus stretch to the four equilibrium stresses defined as those occurring at the end of the loading cycles in Fig. 3. Then, we determined the two constants $\left(k_{0}, M\right)$ by fitting the first two loading cycles of the transient curve. The reasons that we chose not to determine these permeability constants by fitting the transient curve for all cycles or to determine all five constants simultaneously by optimizing the solution to the transient data are the same. In particular, at the apex of the fourth loading cycle in Fig. 3, we would be using our equilibrium stress versus stretch relationship at stretches much larger than those that the relationship is validated for. In other words, the stretch at the apex of the last loading cycle is much larger than the stretch at the end of the fourth loading cycle. If we had optimized the numerical solution to the transient data from all four loading cycles, then we may have used the wrong stress versus stretch curve at this high level of strain, and thus may be optimizing the permeability constants to the wrong quantities. Similarly, if we had attempted to determine all five constants simultaneously by optimizing the solution to the transient data, then we may obtain optimal values of the five constants that, individually, may be incorrect, but collectively produce a good fit to the data in the regions of the ramp phases of the third and fourth cycles where the instantaneous stretches are higher than the final equilibrium stretch. Thus, the method we have used provides a validated fit of the theory to the first two loading cycles and to the four equilibrium stress points.

A final limitation of the present study is that the free energy functions with the determined material constants may not be good approximations to the true free energy functions of the material, because we have only considered experimental data from onedimensional tests whereas the true free energy functions must hold in a higher-dimensional space. Based on our experience of fitting an anisotropic solid strain energy function to annular data $[33,37]$, the strain energy functions used in the present paper will provide large errors when predicting the stress versus stretch response for other deformations. Nevertheless, the reader may use 
the material constants that we have reported in conjunction with the proposed theory to reproduce the mean \pm 1 S.D. response of the annulus to confined compression in an effort to improve upon the estimates of the free energy and permeability functions upon invoking additional experimental data sets.

The physical interpretation of the fluid free energy function is best addressed in the context of the mixture free energy function $\psi$. In Eq. (4), we assumed that $\psi$ can be expressed as an additive decomposition of the constituent free energy functions. For any solid-fluid mixture, it seems reasonable to expect that the mixture free energy function $\psi$ will depend on the nature of both constituents and their interactions. Due to the assumptions made in the special theory, we have assumed that $\psi$ obeys the additive decomposition given in Eq. (10). As such, the manner in which the mixture free energy function $\psi$ depends on the fluid constituent may be modeled by the $\rho^{f} \psi^{f}$ term in Eq. (10), and the manner in which $\psi$ depends on constituent interactions may be modeled in an additive fashion such that these effects would appear in both the $\rho^{f} \psi^{f}$ and $\rho^{s} \psi^{s}$ terms in Eq. (10). It is interesting to point out that Treloar [38] has related the material constants appearing in the theory of finite elasticity to parameters describing the molecular structure of rubbers. In an analogous manner, we may suggest that the material constants appearing in Eq. (10) may be related to parameters describing the molecular structure of cartilaginous tissues, such as proteoglycan content (or fixed charge density), collagen content, the degree of collagen fragmentation, fluid content, and fluid ion concentration. An interaction mechanism that may be modeled by Eq. (10) would include the fixed charge density and the fluid ion concentration, which are intrinsic properties to the solid and the fluid constituents, respectively. Although there exist more structured theories to explain the effect of parameters related to the molecular structure on the mechanical properties of cartilaginous tissues, a mechanical theory of mixtures is certainly desirable for studying certain types of problems and, in a manner analogous to the work of Treloar [38], the material properties appearing in such a theory may be interpreted in terms of the tissue microstructure.

In summary, we have presented an intrinsically incompressible special mixture theory and have determined the material constants for healthy human annulus fibrosus using new confined compression experimental data. In comparison to the results of previous studies, we reported a higher initial water content, a lower aggregate modulus, and a higher initial permeability constant. These differences appear to result from the choice of reference configuration used in the experiments, and we have argued that the reference configuration chosen in the present study is closer to a stress-free reference configuration and, consequently, is more consistent with an assumption made in the constitutive development. Furthermore, we sought to quantify the effect that retaining a particular fluid free energy function has on the solution to the confined compression boundary-value problem using experimental data for the annulus. Our results suggest that the assumption of a constant fluid free energy function is reasonable, as the differences observed in the numerical solutions with and without invoking this assumption are small relative to the inherent tissue variability.

\section{Acknowledgments}

The authors gratefully acknowledge discussions regarding the content of this paper with Professor James Casey (University of California at Berkeley) and the assistance of Frank Ashford, Elisa Bass, and Cari Whyne (Orthopaedic Bioengineering Laboratory, UCSF). Funding was received from UCSF REAC and the National Institutes of Health (AR44179).

\section{References}

[1] Vernon-Roberts, B., and Pirie, C., 1977, "Degenerative Changes in the Intervertebral Discs of the Lumbar Spine and their Sequelae,' Rheumatol. Rehabil., 16, pp. 13-21.
[2] Yasuma, T., 1990, "Histological Changes in Aging Lumbar Intervertebral Discs. Their Role in Protrusions and Prolapses,' J. Bone Jt. Surg., 72A, No. 2, pp. 220-229.

[3] Brickley-Parsons, D., and Glimcher, J., 1984, "Is the Chemistry of Collagen in Intervertebral Discs an Expression of Wolff's Law? A Study of the Human Lumbar Spine,"' Spine, 9, No. 2, pp. 148-163.

[4] Lotz, J. C., et al., 1998, "Compression-Induced Degeneration of the Intervertebral Disc: an in Vivo Mouse Model and Finite-Element Study. 1998 Volvo Award Winner in Biomechanical Studies,' Spine, 23, No. 23, pp. 2493-2506.

[5] Shirazi-Adl, A., Ahmed, A. M., and Shrivastava, S. C., 1986, "A Finite Element Study of a Lumbar Motion Segment Subjected to Pure Sagittal Plane Moments," J. Biomech., 19, pp. 331-350.

[6] Holm, S., and Nachemson, A., 1983, "Variations in the Nutrition of the Canine Intervertebral Disc Induced by Motion,' Spine, 8, No. 8, pp. 866-874.

[7] Urban, J. P. G., et al., 1982, "Nutrition of the Intervertebral Disc,'” Clin. Orthop., 170, pp. 296-302.

[8] Mow, V. C., et al., 1980, 'Biphasic Creep and Stress Relaxation of Articular Cartilage: Theory and Experiments,', ASME J. Biomech. Eng., 102, pp. 7384.

[9] Craine, R. E., Green, A. E., and Naghdi, P. M., 1970, “A Mixture of Viscous Elastic Materials With Different Constituent Temperatures,', Q. J. Mech. Appl. Math., 23, No. 2, pp. 171-184.

[10] Mills, N., 1966, 'Incompressible Mixtures of Newtonian Fluids,' Int. J. Eng. Sci., 4, pp. 97-112.

[11] Armstrong, C. G., and Mow, V. C., 1982, "Variations in the Intrinsic Mechanical Properties of Human Articular Cartilage With Age, Degeneration and Water Content,'” J. Bone Jt. Surg., 64A, pp. 88-94.

[12] Kwan, M. K., Lai, M. W., and Mow, V. C., 1990, “A Finite Deformation Theory for Cartilage and Other Soft Hydrated Connective Tissues-I. Equilibrium Results,'” J. Biomech., 23, pp. 145-155.

[13] Best, B. A., et al., 1994, "Compressive Mechanical Properties of the Human Annulus Fibrosus and Their Relationship to Biochemical Composition,"' Spine, 19, No. 2, pp. 212-221.

[14] Iatridis, J. C., et al., 1998, "Degeneration Affects the Anisotropic and Nonlinear Behaviors of Human Annulus Fibrosus in Compression," J. Biomech., 31, pp. 535-544.

[15] Truesdell, C., and Toupin, R. A., 1960, "The Classical Field Theories," in: Handbuch der Physik, S. Flügge, ed., Springer-Verlag, Berlin.

[16] Green, A. E., and Naghdi, P. M., 1968, “A Note on Mixtures," Int. J. Eng. Sci., 6, pp. 631-635.

[17] Green, A. E., and Naghdi, P. M., 1969, "'On Basic Equations for Mixtures," Q. J. Mech. Appl. Math., 22, pp. 427-438.

[18] Bowen, R. M., 1980, "Incompressible Porous Media Models by Use of the Theory of Mixtures,' Int. J. Eng. Sci., 18, pp. 1129-1148.

[19] Müller, I., 1968, “A Thermodynamic Theory of Mixtures of Fluids," Arch. Ration. Mech. Anal., 28, pp. 1-39.

[20] Ateshian, G. A., et al., 1997, "Finite Deformation Biphasic Material Properties of Bovine Articular Cartilage From Confined Compression Experiments," J. Biomech., 30, No. 11/12, pp. 1157-1164.

[21] Holmes, M. H., and Mow, V. C., 1990, “'The Nonlinear Characteristics of Soft Gels and Hydrated Connective Tissues in Ultrafiltration,'” J. Biomech., 23, pp. 1145-1156.

[22] Holmes, M. H., 1986, "Finite Deformation of Soft Tissue: Analysis of a Mixture Model in Uni-axial Compression,'’ J. Biomech. Eng., 108, pp. 372381.

[23] Oomens, C. W. J., van Campen, D. H., and Grootenboer, H. J., 1987, “A Mixture Approach to the Mechanics of Skin,' J. Biomech., 20, pp. 877-885.

[24] Cohen, B., 1992, "Anisotropic Hydrated Soft Tissues in Finite Deformation and the Biomechanics of the Growth Plate,' Ph.D. dissertation, Columbia University.

[25] Krishnaswamy, S., and Batra, R., 1997, "A Thermomechanical Theory of Solid-Fluid Mixtures,' Math. Mech. Solids, 2, pp. 143-151.

[26] Atkin, R. J., and Craine, R. E., 1976, "Continuum Theories of Mixtures: Applications,"' J. Inst. Math. Appl., 17, pp. 153-207.

[27] Klisch, S. M., 1999, “A Continuum Mixture Theory With Internal Constraints for Annulus Fibrosus,' Ph.D. dissertation, University of California at Berkeley.

[28] Atkin, R. J., and Craine, R. E., 1976, 'CContinuum Theories of Mixtures: Basic Theory and Historical Development,', Q. J. Mech. Appl. Math., 29, pp. 209244.

[29] Klisch, S. M., 2000, "Internally Constrained Mixtures of Elastic Materials,' Mathematics and Mechanics of Solids.

[30] Shi, J., Rajagopal, K., and Wineman, A., 1981, "Applications of the Theory of Interacting Continua to the Diffusion of a Fluid Through Non-linear Elastic Media,' Int. J. Eng. Sci., 19, pp. 871-879.

[31] Thompson, J. P., et al., 1990, "Preliminary Evaluation of a Scheme for Grading the Gross Morphology of the Human Intervertebral Disc,' Spine, 15, No. 5, pp. 411-415.

[32] Madsen, N. K., and Sincovec, R. F., 1979, "Collocation Software for Partial Differential Equations," ACM-TOMS, 5, No. 3, pp. 326-351.

[33] Klisch, S. M., and Lotz, J. C., 1999, “Application of a Fiber-Reinforced Continuum Theory to Multiple Deformations of the Annulus Fibrosus," J. Biomech., 32, No. 10, pp. 1027-1036.

[34] Buschmann, M. D., Soulhat, J., Shirazi-Adl, A., Jurvelin, J. S., and Hunziker, 
E. B., 1998, “Confined Compression of Articular Cartilage: Linearity in Ramp and Sinusoidal Tests and the Importance of Interdigitation and Incomplete Confinement,', J. Biomech., 31, pp. 171-178.

[35] Rajagopal, K. R., and Tao, L., 1995, Mechanics of Mixtures, World Scientific, Singapore.

[36] Reynolds, R. A., and Humphrey, J. D., 1998, "Steady Diffusion Within a
Finitely Extended Mixture Slab," Math. Mech. Solids, 3, pp. 147-167.

[37] Klisch, S. M., and Lotz, J. C., 1999, “Application of a Fiber-Reinforced Continuum Theory to Multiple Deformations of the Annulus Fibrosus,' Adv. Bioeng. ASME, 39, p. 237.

[38] Treloar, L. R. G., 1975, The Physics of Rubber Elasticity, 3rd ed., Clarendon Press, Oxford. 